\title{
Research on Aerodynamic Performance of Ram-air Parafoil's Trailing Edge Deflection
}

\author{
Yanhong Li \\ School of physics \& Electronic Engineering \\ Xian yang Normal University \\ Xianyang 712000, China
}

Xuexia Hua

School of physics \& Electronic Engineering

Xian yang Normal University

Xianyang 712000, China

\author{
Mingjun Wang \\ (1)School of Physics and Electronic Engineering \\ Xian yang Normal University \\ Xianyang712000, China \\ (2) School of Automation and Information Engineering \\ Xi'an University of Technology \\ Xi'an Shaanxi, 710048, China
}

\begin{abstract}
According to the requirement on load and glide slope of parafoil in practical engineering, the parameters of aspect ratio, span and anti-arcangle are computed and designed based on the known two-dimensional airfoil cross-section of parafoil. Firstly, with finite volume method to solve the NavierStokes control equation of K-epsilon model, performances of three-dimensional structure of parafoil, which was tested in NASA Langley research center to validate the feasibility of the method. The same method is applied to the designed parafoil to obtain the aerodynamic coefficients under different attack angles and different trailing edge of deflection, which provide basis for building the kinetics model accurately.
\end{abstract}

Keywords-parafoil; parameter design; aerodynamic performance; trailing edge deflection

\section{INTRODUCTION}

Compared to conventional parachute, ram-air parafoil is widely used in the military field of recycling and return of aircrafts. With its superior controllability and gliding performance, parafoil reduces the possible landing area and improves the accuracy of the landing point. Meanwhile, due to the excellent maneuverability and reliability, the conventional parachute is gradually replaced by modern parafoil which is favored by parachuting athletes. More concentration is focused on controllable large ram parafoil, at the same time, it fastens the pace of simulations and validation of experiments.

The aerodynamic performance research on ram-air parafoil began earlier and developed better abroad from the 1960's, and experienced from the stage of airdrop tests and tunnel experiments to the numerical simulation. NASA Langley Research Center had studied the change of aerodynamic coefficients such as lift coefficient and drag coefficient under different angle of attacks, which has provided a large amount

AUTHOR: Li Yanhong (1983-), female, postgraduate.

Our works is This works is partly supported by the National Natural Science Foundation of China (Grant No. 61771385), Key industry innovation chain of Shaanxi Province(Grant No.2017ZDCXL-GY-06-01)and scientific project of Xian Yang Normal University (NO.10XSYK310). of data for the validation of further numerical simulation[1]. Uddin had studied the pressure distribution on the up and lower surface and the associated aerodynamic characteristics, meanwhile the impact that cutter of leading edge has on the parafoil properties is studied[2]. Desabrais selected the airfoil of flat lower surface, which is applied in airdrop test system, and studied the flow field of two kind of leading edge shapes, what's more important, the effect of unsymmetrical trailing edge deflection to lateral motion and symmetrical trailing edge deflection to longitudinal motion were analyzed, at last, the result of simulation and wind tunnel tests were compared[3]. Bergeron has built the dynamic equilibrium model between the structure and fluid by the developed commercial software of LS-DYNA under the condition of three-dimensional model, at the same time, the process of canopy inflation and the flow filed were studied[4].

Compared to foreign countries, domestic research works about parafoil began late and it exists a big gap. Nanjing University of Aeronautics and Astronautics, China Aerodynamics Research and Development Center conducted wind tunnel tests of parafoil in order to provide theoretical basis and optimization strategies for the design, and clarified the mechanism. Recent years, with the advancement of computer technology, the domestic numerical simulation develops rapidly. Li Jian computed the aerodynamic forces of the two-dimensional airfoil section profile, and studied how the cutter angle and cutter height, affected the aerodynamic characteristics [5]. Experiment were based on the parafoil adopted by NASA Langley Research Center, without considering the flexibility of canopy ,conducted the numerical simulation on different shape parafoils which have air chambers with aspect ratio of 3[6][7]. Li Yang studied the performances of parafoil when the trailing edge deflection acted on the system, however, it mainly concentrated on the two-dimensional airfoil section simulation, ignored the comparison with unsymmetrical and symmetrical trailing edge deflection[8] .

In this article, the steady gliding properties is studied, on account of we only need to research the steady performance of 
the system, we can ignore the inflation of canopy. Firstly, based on the modified airfoil section, the structure of parafoil is designed according to the requirement. Secondly, numerical simulation under the condition of unsymmetrical and symmetrical trailing edge deflection is conducted, by comparing the two situations, we can derive how the aerodynamic properties changes with the amount of deflection, and the accurate coefficients are vital for kinetic modeling.

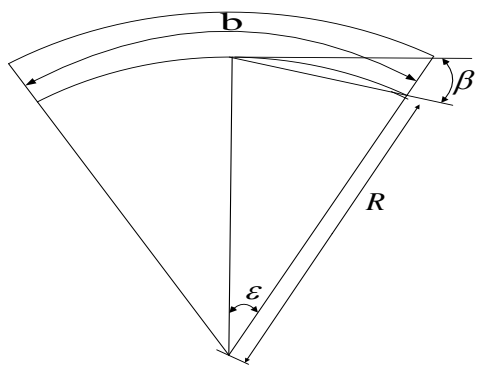

Fig. 1. Front view of the structure of parafoil

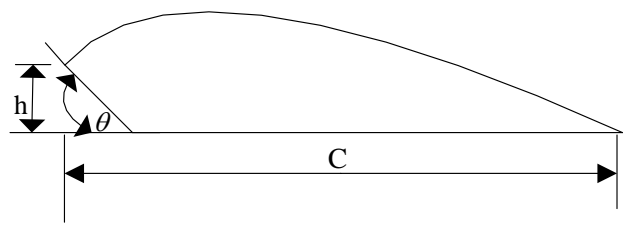

Fig. 2. The airfoil section with leading edge cutter

\section{DESIGN OF PARAMETERS}

This paper mainly studies the aerodynamic performance of steady gliding stage of the parafoil system which ignores the effects of load and parafoil suspension lines acting on the aerodynamic characteristics. It focuses on the specific design and calculation of the parameters of the canopy.

A simplified structure of parafoil is showed in Fig.1 , and the parafoil can be seen as a period of circular arc when it is inflated, The radius of the round is $\mathrm{R}, \mathrm{b}$ refers to the span of the canopy and is the length of circular arc, $\beta$ represents the anti-arc angle ,and $\varepsilon$ is half of the central angle according to the circular arc. The two-dimensional airfoil section with leading edge cutter is represented in Fig.2, c is the length of the chord, $h$ represents the height of cutter and $\theta$ is defined by the angle between lower surface and cutter line.

\section{A. Selection of airfoil profile}

In the process of parafoil design, the selection of the airfoil is important to the whole process, which directly affects the aerodynamic performance of the parafoil system. Generally, parafoil works at low speed, so we select the low speed airfoil, that has round nose and tip trailing edge. Clark-Y airfoil section is of simplified shape, which has superior lift-to-drag force characteristics and is widely used in the design of parafoil. Clark-Y is selected as the basic airfoil profile, if the selection of cutter height ranges from $40 \%$ to $50 \%$ of the maximum thickness of the airfoil profile, the aerodynamic performance will be better and it is detrimental to inflation if the angle and height of the cutter are less. however, it is beneficial to inflate and reduce the glide ratio as the opposite condition occurs. In this article, we choose $45 \%$ of the maximum thickness as the cutter height.

\section{B. Aspect ratio}

The aspect ratio is defined by ratio of the span of the canopy b to the chord c. For a given angle of attack, the bigger the aspect ratio, the higher lift coefficient and lower drag coefficient, so absolutely the glide ratio increases with the aspect ratio increasing, the higher aspect ratio can bring good aerodynamic performances. However, large aspect ratio would reduce the parafoil rigidity and makes the hanging system too large to control. According to the design experience, the aspect ratio in this article is taken as 3 .

\section{Plane shape of the canopy}

There are two main kinds of commonly used plane shape of canopy, one of which is rectangular airfoil of same chord length spanwise, the other is oval airfoil of thicker central chord. In this paper, the main consideration is to process the parafoil easily, so we choose the rectangular shape of canopy.

\section{The chord and span}

According to the rectangular shape and the area of the canopy, we can obtain

$$
\begin{gathered}
A_{s}=b^{*} c \\
\lambda=\frac{b}{c}=\frac{b^{2}}{A_{s}}
\end{gathered}
$$

On the above equations, $\lambda$ refers to aspect ratio, $A_{s}$ refers to area of the canopy. In this paper, we choose $b=2.45 \mathrm{~m}$, $c=0.82 \mathrm{~m}$.

\section{E. The anti-arc circular angle}

Anti-arc angle will affect the stability and aerodynamic performance of the parafoil system. Increasing the anti-arc circular angle will lead to the decrease of effective lift and lower aerodynamic performance, the small anti-arc angle results in the decrease of lateral stability. In engineering, for the parafoil with small aspect ratio, the range of anti-arc is within $10^{\circ}$, for the parafoil with large aspect ratio, we can increase the anti-arc angle properly, it is about 30 . According to the engineering experience, the following equation shows the relation of lift coefficient and anti-arc angle [9].

$$
C_{\mathrm{y}}=C_{y, \beta=0} * \cos ^{2} \beta
$$

Form the above equation, when the anti-arc angle reaches , lift coefficient decreases to the $3 / 4$ of the original where the anti-arc angle is $0^{\circ}$. When the aspect ratio is large but the anti -arc angle too small, it is necessary to lengthen the suspension lines, which may lead to the deformation of the canopy. Here we select the anti-arc angle as $20^{\circ}$.

\section{NUMERICAL SimULATION RESUlTS}

\section{A. Conditions}

In this paper, the calculation of the canopy model using the following simplifying assumptions:

1) Treat parafoil canopy as a rigid body, regardless of its aerodynamic deformation. 
2) The air permeability amount of airfoil profile is zero.

3) Parafoil speed is very low, so it is assumed to be incompressible flow.

4) Suppose the fluid as Newtonian fluid.

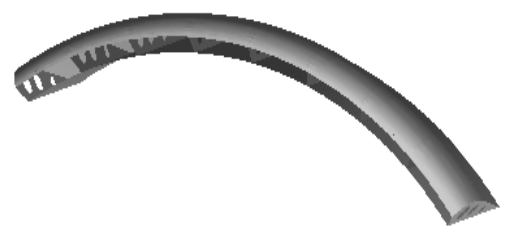

Fig. 3. Three-dimensional structure figure of model

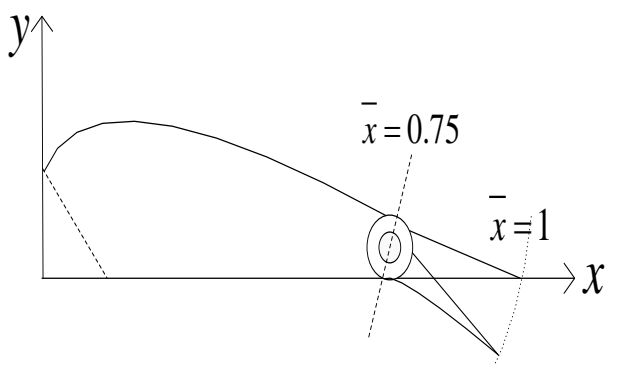

Fig. 4. Airfoil profile of trailing edge deflection

\section{B. Grid partition}

The structural figure of parafoil is showed in Fig.3, and meshing is based on ANSYS software. Establish the rectangular flow filed, the size of which is $16 c * 10 c * 10 c$. Choose the angle of attack as $-4^{\circ} 、 0^{\circ} 、 4^{\circ} 、 6^{\circ} 、 8^{\circ} 、 10^{\circ} 、 12^{\circ} 、 14^{\circ}$, divide grid of the model under selected angles of attack separately.

The method of bottom-up meshing division is adopted, the entire grid is tetrahedral, the first step is to create surface grid, then advancing front method is used to generate body grid automatically. The flow filed around the parafoil model varies intensely, in order to descript it accurately, the densified mesh is generated by creating the rectangular density box, away from the model, and the mesh can be sparser to reduce the amount of calculation.

\section{Aerodynamic performances of designed parafoil}

The spiral and flare movement of parafoil system are achieved through the trailing edge deflection in different degree, the definition of edge deflection adopted in the paper is same with the wind tunnel tests. Tests model bends at the $75 \%$ chord length used as shaft. The deformation on the front of canopy is small, only the rear $25 \%$ of the wing area is deflected.

Airfoil profile of trailing edge deflection is showed in fig.4. When there is symmetric trailing edge deflection, the angle of bending along span direction is consistent; when there is unsymmetrical trailing edge deflection, the degree of side bending is same to the case of symmetric deflection, and the bending reduces until to the symmetric plane where the deflection is 0 . Model is simulated at different flap deflection condition defined as $0,1 / 6,1 / 3,1 / 2$ in fraction of the whole chord length. The bending angles corresponding to the deflection is showed in the table1.

TABLE I. RELATION BETWEEN DEFLECTIONS OF TRAILING EDGE AND BENDING ANGLE

\begin{tabular}{cc}
\hline Deflection & Bending angle $\left({ }^{\circ}\right)$ \\
\hline 0 & 0 \\
\hline $1 / 6$ & 12.5 \\
\hline $1 / 3$ & 25 \\
\hline $1 / 2$ & 37.5
\end{tabular}

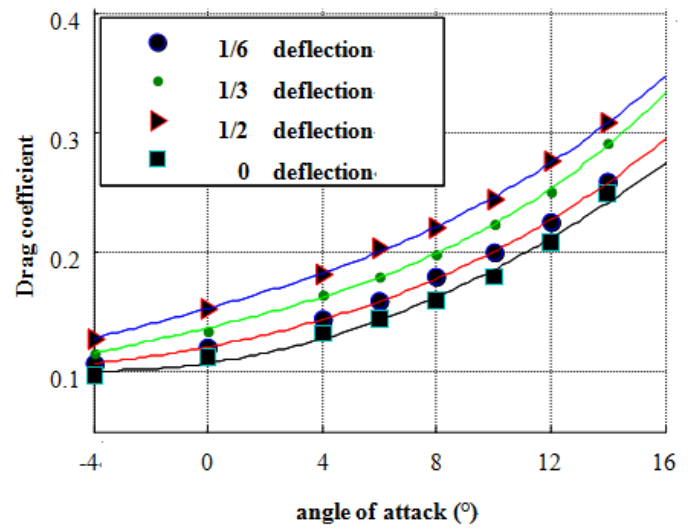

Fig. 5. Drag coefficient under different unsymmetrical trailing edge deflection

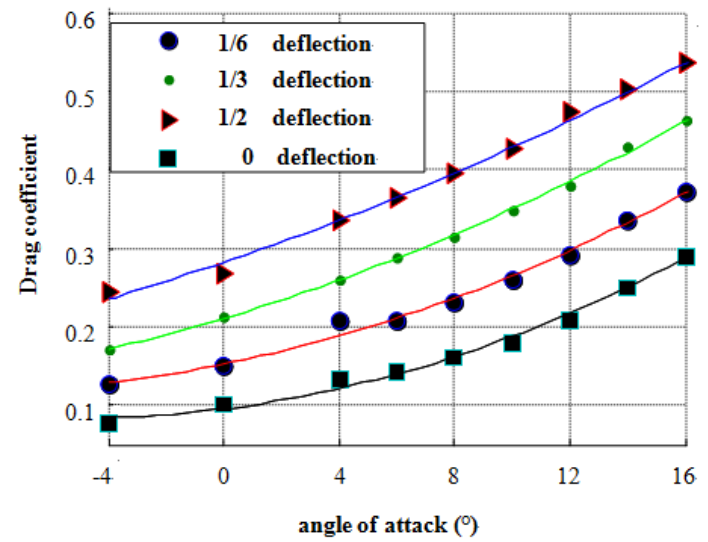

Fig. 6. Drag coefficient under different symmetrical trailing edge deflection

For grid division and boundary condition, adopt the same setting as the wing trailing edge without deflection, it seems verbose to mention the grid division and conditions setting, the numerical simulation results are given directly.

Fig.5 and Fig.6 above show the trend of drag coefficient changing with angle of attack under unsymmetrical and Symmetric trailing edge deflection, the larger deflection, the 
greater drag coefficient, the drag coefficient under deflection is larger than that of no deflection, the drag coefficient under symmetric deflection is larger than unsymmetrical deflection.

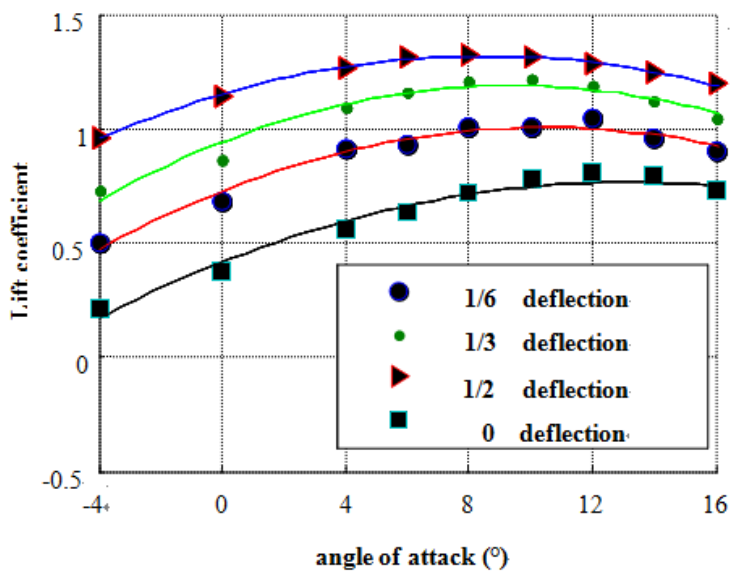

Fig. 7. Lift coefficient under different unsymmetrical trailing edge deflection

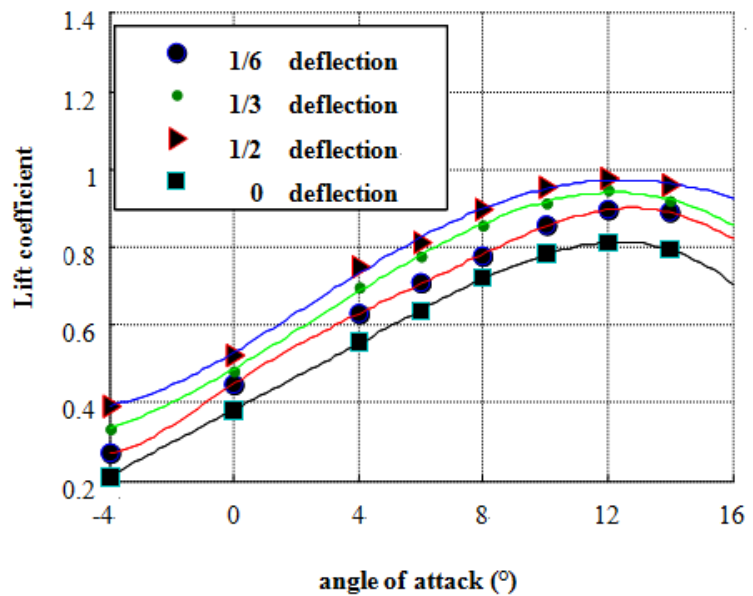

Fig. 8. Lift coefficient under different symmetrical trailing edge deflection

Fig. 7 and Fig.8 above show the trend of lift coefficient changing with angle of attack under unsymmetrical and Symmetric deflection, under the same condition, the lift coefficient corresponding to symmetric deflection is larger than that under the same amount of unsymmetrical deflection.
When there is unsymmetrical deflection of the trailing edge, the lift coefficient increases first, and then decreases, the trend is consistence with that no deflection. The stall angle of attack appears at around , similar to the case of zero defection.

\section{CONCLUSION}

In this paper, the important parameters of parafoil were designed, the aerodynamic performance according to unsymmetrical and symmetric deflection of trailing edge was studied, no matter on unsymmetrical or symmetric deflection, lift coefficient and drag coefficient increased with the angle of attack on the rise, however, the lift-to-drag ratio declined to some degree.

\section{REFERENCES}

[1] Nicolaides J D, "Parafoil wind tunnel tests," Notre Dame Univ in Dept of Aerospace and Mechanical Engineering, 1971.

[2] Uddin M N and Mashud M, "Wind tunnel test of a paraglider (flexible) wing canopy," International Journal of Mechani-cal:-Mechatronics Engineering, Vol. 10(3), pp: 7-18, 2010.

[3] Desabrais K J, Bergeron K, Nyren D, and Johari H, "Aerodynamic Investigations of a Ram-Air Parachute Canopy and an Airdrop System," 23rd AIAA Aerodynamic Decelerator Systems Technology Conference, pp: 2145, March 2015

[4] Bergeron K, Seidel J, and McLaughlin T. "Wind Tunnel Investigations of Rigid Ram-Air Parachute Canopy Configurations,” 23rd AIAA Aerodynamic Decelerator Systems Technology Conference, pp: 2156, 2015

[5] Li Jian, "Leading Edge Cutter on Aerodynamic Performance,” Journal of air return and remote sensing,Vol. 26(1), pp:36-41, 2005. (In Chinese)

[6] Zhu Xu, and Cao Yihua, "The Effect of Parafoil Plane Shape on Aerodynamic Performance," Journal of aviation, Vol. 32 (11), pp:19982007, 2011. (In Chinese)

[7] Zhu Xu, and Cao Yihua, "The Impact of Anti-arc Angle, Airfoil Profile and Leading Edge Cutter on Parafoil Aerodynamic Performance," Journal of aviation, Vol. 33 (7), pp: 1189-1200, 2012. (In Chinese)

[8] Li Yang, "Ram-air Parafoil Trailing Drop-down Features of Numerical Research," Changsha: National University of Dense Technology, 2004.(In Chinese)

[9] Fogell, N, Sherwin, S, Cotter, C. J, Iannucci, L, Palacios, R, and Pope, D J, "Fluid-Structure Interaction Simulation of the Inflated Shape of RamAir Parachutes,” 22th AIAA Decelerator Systems (ADS) Conference, Daytona Beach, Florida, March 2013. 